\title{
Figement de séquences défigées
}

Un commerce devenu inéquitable

\section{Christophe Cusimano}

\section{(2) OpenEdition \\ Journals}

\section{Édition électronique}

URL : http://journals.openedition.org/pratiques/2833

DOI : $10.4000 /$ pratiques.2833

ISSN : 2425-2042

Éditeur

Centre de recherche sur les médiations (CREM)

\section{Édition imprimée}

Date de publication : 15 décembre 2013

Pagination : 69-78

\section{Référence électronique}

Christophe Cusimano, «Figement de séquences défigées », Pratiques [En ligne], 159-160 | 2013, mis en ligne le 30 juin 2016, consulté le 20 avril 2019. URL : http://journals.openedition.org/pratiques/2833 ; DOI : 10.4000/pratiques.2833 


\title{
Figement de séquences défigées. Un commerce devenu inéquitable ${ }^{(*)}$
}

\author{
Christophe Cusimano
}

Université Masaryk de Brno ${ }^{(1)}$

\section{Du devenir des défigements}

La sémantique componentielle s'est constituée à partir d'analyses de décomposition des unités significatives. Or, dès qu'il faut envisager le « contexte » et replacer les unités dans leur milieu naturel si l'on peut dire, le manque d'outils s'y fait criant. Il faut alors ajouter de nouvelles règles pour décrire tel nouveau phénomène contextuel. Si à l'inverse, on envisage la langue comme un espace de liberté relatif, il faut peut-être admettre que c'est le global qui prend le dessus sur les unités de la langue et les règles qui semblent les accompagner.

Dans certains cas, l'existence même de la distinction entre morphème et lexie se trouve conditionnée par des conditions herméneutiques et donc exo-lexicales. Comme le dit F. Rastier (1997: 310),

« Le découpage morphologique résulte de parcours interprétatifs et dépend de conditions herméneutiques. Ainsi, l'expression monter au créneau comprend trois lexies dans Bayard monte au créneau, mais une seule dans Rocard monte au créneau. Naturellement, aucune grammaire n'admettrait la règle : si Rocard apparaît dans le contexte de monte au créneau, alors monte au créneau compte pour une lexie».

Les défigements de séquences figées (ces idiomatismes qui sont partout dans la langue au point de représenter un quart à un tiers du lexique d'une langue) sont particulièrement emblématiques de cette prédominance du global sur le local, comme le montre l'exemple ci-dessus. L'objet de cet article n'est en aucune ma-

(*) Une version préliminaire de cet article a été publié en 2012 sous format numérique dans : Christophe Cusimano (2012) «Figement de séquences défigées », [En ligne], Volume XVII, n4 (2012). Coordonné par Audrey Moutat, URL : http://www.revuetexto.net/index.php?id=3105

(1) Pour toutes questions, suggestions, remarques : ccusim@phil.muni.cz 
nière de revenir sur les propriétés des séquences figées ${ }^{(2)}$ ou figements ${ }^{(3)}$, ni de discuter les degrés d'intégration des morphèmes qui les composent par exemple : un grand nombre de travaux balisent déjà fort bien ce champ d'étude, miné d'exceptions dues au fait que le locuteur reste libre de maltraiter toute séquence figée et d'emprunter un parcours interprétatif inexploré. Toutefois, précisons que nous nous accordons avec B. Fradin (2003 : 220-221) lorsqu'il écrit ces lignes :

« Suivant Corbin (1992), je considère que la lexicalisation est le fait qu'une expression linguistique accède au statut d'entité codée, présentant une association réglée des rapports son / sens / syntactique reconnue et employée comme telle. Avec Bauer (1983, 48-61), on peut la voir comme la dernière étape d'un processus qui se marque par le fait qu'une expression devienne une unité lexicale en acquérant au moins une propriété idiosyncrasique ».

Nous rejoignons aussi A. Krieg-Planque (2012 : 99-100) qui, d'un point de vue non plus morphologique mais discursif décrit le figement « comme espace de resserrement de la créativité verbale » : en effet, écrit-elle plus loin (2012:103), « la succession d'unités prévisibles sur la chaîne verbale engendre un effet de fermeture du discours sur lui-même, l'impression qu'il n'est pas aisé, voire pas prévu, de trouver une place dans un discours qui apparaît en quelque sorte comme « déjà prévu » ». Cette dimension, nous le verrons, est essentielle pour les défigements que nous aurons à traiter.

Mais dans cet article, plutôt que revenir sur ces aspects succinctement évoqués, nous projetons de mesurer le devenir des défigements, de formuler des hypothèses sur les conditions qui conduisent à un nouveau figement, celui de l'expression défigée qui va donc coexister avec le premier figement. C'est le cas de «commerce inéquitable ", défigement formé par ajout du préfixe " in- " à la séquence figée « commerce équitable». Une telle expression a sans doute acquis à son tour le statut de lexie, puisqu' on en recense pas moins de 20.000 attestations sur Google.fr (contre 788.000 au figement premier). D'ailleurs, dans cette étude, nous allons nous intéresser en grande partie aux défigements produits par ajout d'affixe à l'un des éléments de la séquence figée : ce parti-pris, plus encore que limiter le nombre de figements retenus, permet peut-être de cibler plus aisément ceux qui sont susceptibles de former par leur caractère bref une formule ${ }^{(4)}$ à nouveau lexicalisable. On comprend dès lors que l'objet de cet article est de voir si, parmi des défigements produits selon un même mode opératoire, il est possible de prédire lesquels ont des chances de devenir eux-mêmes lexies. Car s'il est admis que « les défigements redonnent donc vigueur à des locutions, à des mots composés auxquels, habituellement, chacun accorde peu d'importance » (B. Habert et P. Fiala, 1989: 86 ), on cherche trop rarement à associer prédictions et étude de veille lexicale.

En d'autres termes, nous cherchons à étayer, par l'observation de certaines régularités et la formulation de certaines hypothèses prédictives, l'option définie par A. Krieg-Planque (2012: 105) comme l'étude des « modalités formelles de cir-

(2) Ou SF. Nous reprenons ici la terminologie employée par P. Fiala et B. Habert (1989)

(3) On rencontre en anglais les termes MWEs (multi words expressions), FEIs (fixed expressions and idioms), ou encore set phrases.

(4) Nous employons le terme dans le sens que lui attribue A. Krieg-Planque (2009: 7 ); « un ensemble de formulations qui, du fait de leurs emplois à un moment donné et dans un espace public donné, cristallisent des enjeux politiques et sociaux que ces expressions contribuent dans le même temps à construire ». Cet article en fait aussi ressortir la dimension satirique, ou du moins ludique. 
culation des énoncés que les acteurs sociaux co-produisent et identifient comme étant des slogans ».

\section{Quelques défigements par ajout segmental}

Signalons d'emblée que l'ajout d'un affixe à l'un des segments de la séquence figée est, dans les exemples qui suivent (sauf(h) peut-être), sans effet syntaxique : ce procédé s'apparenterait donc plutôt, sans en être, à de la dérivation, même si bien sûr, la dérivation peut avoir un effet syntaxique d'un point de vue catégoriel. Il est clair aussi que dans tous ces cas l'ajout ne fait pas du segment concerné un mot nouveau. C'est pourquoi parler d'affixation (dans le sens productif) n'est pas parfaitement adéquat non plus, puisque ce procédé n'a d'autre motivation qu'une ambition ludique, tout du moins dans le contexte du corpus majoritairement satirique parcouru ici : en effet, ce corpus, perçu comme un préalable à une recherche de plus grande envergure, concentre des faits de langue - ou plutôt de discours qui investissent l'énonciation grâce à une série d'enjeux ciblés : innovation sémantique, calembours, discours journalistique, enjeux sociopolitiques, etc. Ainsi se profilent des entités prenant des formes que l'on ne trouvera pas nécessairement dans d'autres corpus. Mais il faudrait alors aussi se demander ce qu'est un corpus « standard» : la presse classique, dont on traite le plus souvent, n'obéit-elle pas à des codes fixes et tout aussi particuliers ?

Tout en gardant ceci à l'esprit à propos de notre corpus, nous nous contenterons de parler ici d'ajout segmental, ce qui permet d'englober à la fois ce que F. Rastier appelle les défigements par intercalation $(\mathrm{f})^{(5)}$, et les cas où le défigement est produit par l'ajout d'un préfixe (a, c, d, e) ou d'un suffixe (b), (g) qui se présente comme un défigement basé sur le signifiant, et (h) que l'on range généralement parmi les défigements produits par ajout non-morphologique.

En ce qui concerne les défigements relevés sur le site Cahiers du football.fr, on peut noter qu'ils sont la plupart du temps contenus dans la partie gauche des titres bisegmentaux à deux points - soit en premier : ceci vise peut-être à mettre en avant leur statut de formule, acquis ou à acquérir. L'interprétant se trouverait donc souvent à droite.

(a) ... celui d'un port nanti de tous les engins les plus modernes, qu'ils se firent construire sur démesure. (Le Canard Enchaîné du 24.08.2011).

(b) David Douillet, catégorie poids lourdingue (et médaille d'or du fayotage sarkozyste) (Le Canard enchainé du 08.10.2011).

(c) Cible émouvante : Benjamin Gavanon (L'Équipe) : « Moi j’ai pris une baffe de Bagayoko » (Cahiers du football.fr).

(d) Réanimation offensive ${ }^{(6)}$ : José Mourinho (lequipe.fr) : «Benzema n'est pas mort ». (Cahiers du football.fr)

(e) Commerce inéquitable ${ }^{(7)}$ : «Transferts : échange ASSE-Juve ? (sport24.fr). (Cahiers du football.fr).

(5) Il est vrai que l'on parle habituellement d'intercalation seulement dans les cas où un segment est inséré entre les unités de la SF, alors qu'ici, c'est entre les deux syllabes d'un lexème.

(6) "Animation offensive ", dans le jargon footballistique, désigne la capacité d'une équipe à mettre en place son jeu d'attaque.

(7) Nous conviendrons volontiers que dans ce contexte footballistique, il ne s'agit pas tout à fait de la «même » lexie "inéquitable » que dans « commerce inéquitable et petits producteurs » par exemple, où il s'agit d'une critique économique et politique du commerce dit «équitable »mais ne l'est pas. 
(f) Et (par enchantement ?) les occasions d'inscrire un but arrivent enfin pour l'Olympique de Marseille, pas de quoi s'enflammer non plus, surtout avec un Samassa devant, le «vendangeur masqué » (Hors-jeu passif.unblog.fr).

(g) Défaite de la bière : "Allemagne, la peur du bide » (Cahiers du football.fr).

(h) Strident ${ }^{(8)}$ offensif : "Toulouse : Larsen dans le groupe » (Cahiers du football.fr).

(i) Ampère Fouettard : "Ancelotti veut "plus d'intensité" » (lequipe.fr) (Cahiers $d u$ football.fr).

Quelles sont les raisons qui font que certaines de ces expressions défigées se lexicalisent pourvues de l'ajout en question, morphologique ou non ? Il est bien évident que le faible nombre de défigements isolés ${ }^{(9)}$ ne permettra pas de formuler plus que des hypothèses. L'objectif revêt toutefois un intérêt certain que le tableau suivant doit permettre de mieux cerner. Y prennent place tous les défigements étudiés que nous commentons séparément ci-après.

\begin{tabular}{|c|c|c|c|c|c|c|}
\hline & SF & SF défigées & Procédé & $\begin{array}{l}\text { Indices } \\
\text { interprétatifs } \\
\text { pour } \\
\text { la présomption } \\
\text { d'isotopie }\end{array}$ & $\begin{array}{l}\text { Relation entre la SF } \\
\text { et la SF défigée }\end{array}$ & $\begin{array}{l}\text { Attestations de } \\
\text { la SF et de la } \\
\text { SF défigée }\end{array}$ \\
\hline$a$ & $\begin{array}{l}\text { sur } \\
\text { mesure }\end{array}$ & $\begin{array}{l}\text { sur } \\
\text { démesure }\end{array}$ & $\begin{array}{l}\text { ajout de } \\
\text { préfixe }\end{array}$ & $\begin{array}{l}\text { «les plus } \\
\text { modernes » }\end{array}$ & $\begin{array}{l}\text { mise en opposition sur } \\
\text { un plan évaluatif }\end{array}$ & $\ldots{ }^{(10) / 2.240}$ \\
\hline b & $\begin{array}{l}\text { poids } \\
\text { lourd }\end{array}$ & $\begin{array}{l}\text { poids } \\
\text { lourdingue }\end{array}$ & $\begin{array}{l}\text { ajout de } \\
\text { suffixe }\end{array}$ & « fayottage » & $\begin{array}{l}\text { péjoration à l'aide } \\
\text { du suffixe et } \\
\text { resémantisation de } \\
\text { « lourd » }\end{array}$ & $\ldots / 800$ \\
\hline c & $\begin{array}{l}\text { cible } \\
\text { mouvante }\end{array}$ & $\begin{array}{l}\text { cible } \\
\text { émouvante }\end{array}$ & $\begin{array}{l}\text { ajout de } \\
\text { préfixe }\end{array}$ & «pris une baffe» & $\begin{array}{l}\text { changement de do- } \\
\text { maine : sème mésogéné- } \\
\text { rique } \\
\text { différent }\end{array}$ & $62.000 / 23.000$ \\
\hline d & $\begin{array}{l}\text { animation } \\
\text { offensive }\end{array}$ & $\begin{array}{l}\text { réanimation } \\
\text { offensive }\end{array}$ & $\begin{array}{l}\text { ajout de } \\
\text { préfixe }\end{array}$ & «pas mort» & changement de taxème & $146.000 / 5$ \\
\hline e & $\begin{array}{l}\text { commerce } \\
\text { équitable }\end{array}$ & $\begin{array}{l}\text { commerce } \\
\text { inéquitable }\end{array}$ & $\begin{array}{l}\text { ajout de } \\
\text { préfixe }\end{array}$ & $\begin{array}{l}\text { lien } \\
\text { «ASSE-Juve » }\end{array}$ & négation de l'équité & $788.000 / 20.000$ \\
\hline f & $\begin{array}{l}\text { vengeur } \\
\text { masqué }\end{array}$ & $\begin{array}{l}\text { vendangeur } \\
\text { masqué }\end{array}$ & intercalation & $\begin{array}{l}\text { « surtout avec } \\
\text { Samassa } \\
\text { devant » }\end{array}$ & $\begin{array}{l}\text { mise en opposition : le } \\
\text { sauveur devient le } \\
\text { fossoyeur }\end{array}$ & $163.000 / 22.700$ \\
\hline$g$ & $\begin{array}{l}\text { fête de la } \\
\text { bière }\end{array}$ & $\begin{array}{l}\text { défaite de } \\
\text { la bière }\end{array}$ & $\begin{array}{l}\text { ajout de } \\
\text { préfixe permis par } \\
\text { l'homophonie } \\
\text { «fête »/« faite» }\end{array}$ & « bide » & $\begin{array}{l}\text { opposition euphorique } \\
\text { /dysphorique }\end{array}$ & $278.000 / 4$ \\
\hline h & $\begin{array}{l}\text { trident } \\
\text { offensif }\end{array}$ & $\begin{array}{l}\text { strident } \\
\text { offensif }\end{array}$ & $\begin{array}{l}\text { ajout non-mor- } \\
\text { phologique }\end{array}$ & « Larsen » & $\begin{array}{l}\text { phonique : une attaque } \\
\text { bruyante mais inefficace }\end{array}$ & $58.000 / 5$ \\
\hline
\end{tabular}

(8) Un « trident offensif » est une attaque à trois joueurs, trois « pointes » comme on peut aussi le dire.

(9) Aucune méthode automatique ne permet de le faire. Toutefois, à notre avis, cette liste contient de nombreux cas prototypiques : ainsi, les remarques faites au sujet de "commerce équitable/inéquitable » peuvent être transposées à d'autres cas, comme celui de « (les) responsables/irresponsables politiques », en exceptant la différence de nature grammaticale bien sûr.

(10) Les « ...» marquent un nombre non-calculable. 
Sans dévoiler ici l'essentiel de notre propos, revenons rapidement sur ces exemples de défigements : deux cas, (a) et (g) marquent une opposition à l'aide du préfixe "dé ", puisque "défaite ", sans être bien sûr le pendant contraire de « faite » (pas plus que de « fête » toutefois convoqué ici) comme « démesure » est celui de "mesure », produit le même effet du point de vue du signifiant : par ailleurs, même au regard des parcours interprétatifs, dans les deux cas c'est une opposition euphorique/dysphorique qui est ainsi mise en valeur. La relation est plus complexe pour «démesure »dans le sens où l'évaluation doxale est en jeu : c'est bien le point de vue de l'énonciateur qui se trouve mis au premier plan. Au niveau quantitatif, le thème du bierbauch qui marque la « défaite de la bière », signalé par «bide» et «bière», ne recueille que 4 suffrages quand la folie des grandeurs dite « sur démesure » (ici sur l'île de Nauru) semble plus communément répandue. La resémantisation de « lourd » dans « poids lourdingue », produite par ajout de suffixe et une nette présomption d'isotopie sur la base de «fayottage», conduisent à une péjoration évidemment inexistante dans « poids lourd ». Le préfixe «é- » dans « cible émouvante »indique une distance avec l'isotopie /militaire/, par l'appartenance de «émouvante » au taxème //sentiments//. Toujours selon ce procédé, notons le changement de taxème par l'ajout de « ré- » à « animation » (//sport//), puisque « réanimation » renvoie à la //médecine//: on pourrait même dire qu'on change de lexie et la nouvelle lexie n'appartient pas au même domaine («animation »/sport/ conduit à « réanimation »/médical/). Ainsi, « mort» est à entendre non pas au sens d'un joueur à bout de souffle mais, littéralement, à sauver. Tout comme pour «bide » (g), il s'agit ici d'une syllepse. Pourquoi le commerce dans (e) est-il qualifié d' « inéquitable » ? Par afférence, bien sûr guidée par les interprétants, si l'on sait que les deux clubs St.-Étienne et Turin ne sont pas considérés comme étant de même niveau. Restent enfin trois cas d'ajouts non-morphologiques, (f), (h) et (i) : dans le premier, on pourrait éventuellement parler de mise en opposition, puisqu' on dit parfois d'un buteur qu'il «venge» son équipe; le «vendangeur » ${ }^{(11)}$, au contraire, gâche les occasions de but qui s'offrent à lui. Quant au second, il matérialise le passage d'une attaque à trois têtes à un effet Larsen suggérant que son équipe ne se portera pas mieux en sa présence. Enfin, ledit père fouettard en (i), en l'occurrence l'entraîneur de l'équipe, semble ne plus administrer de coups de martinet mais des décharges électriques. Aucune opposition n'y est donc mise en évidence.

\section{Facteurs de re-figement}

Avant d'essayer de déterminer pourquoi certains défigements se figent à leur tour, il faut sans doute déterminer à partir de quand il y a re-figement. En ce sens, l'une des options les plus pertinentes porte sur 1'exploitation de la dernière colonne du tableau : le nombre d'attestations des différentes séquences, qui ont été relevées sur Google.fr, semble permettre dans la plupart des cas de tracer la limite entre défigements occasionnels et défigements en voie de figement. Les cas les plus clairs concernent pour la première catégorie $(\mathrm{d}),(\mathrm{g})$ et $(\mathrm{h})$, pour la seconde (a), (c), (e) et (f) ; seul (b) reste incertain mais semble aussi engagé dans le processus. Essayer toutefois de dire à partir de quel nombre d'attestations une séquence est figée n'est pas aisé. Peut-être est-il plus pertinent d'évoquer un ratio SF/SF

(11) Même si «vendangeur masqué », hors de la sphère footballistique, possède encore un autre sémantisme. 
défigée : ainsi, dans le cas de « commerce équitable» / « commerce inéquitable», on aurait un ratio de 1/40 environ en faveur du premier, contrairement à moins de $1 / 3$ pour « cible mouvante » / " cible émouvante », mais cela est surtout dû au fait que le défigement est aussi le titre d'un film. Cette voie n'est donc satisfaisante qu'à condition d'éliminer les résultats identiques, ce que les moteurs de recherche permettent généralement de faire. Auparavant, il convient toutefois de les avoir identifiés tous.

En venant à présent aux facteurs qui pourraient peser dans le sort réservé aux défigements produits par ajout segmental, il paraît envisageable d'évoquer un critère sémantique : le fait que ceux-ci soient liés aux séquences figées originelles par une relation d'opposition, de négation, de distanciation, s'impose comme une option crédible de ce point de vue. Peut-être est-ce aussi lié au fait que dans les cas de « démesure » et de « inéquitable», ce n'est pas la duplicité sémantique et le côté ludique qui prédominent mais bien l'effet de sens en lien avec la prise de position de l'énonciateur, en d'autres mots la modalité évaluative ou le plan dialogique, le point de vue de l'énonciateur dans un monde contrefactuel.

Outre l'épreuve des faits, comme en attestent « commerce inéquitable », «vendangeur masqué » et « sur démesure », qui sont plus ou moins courants désormais, on peut penser que le défigement contribue efficacement à l'élaboration de formules antonymes, ou du moins de formules dont la seconde nie la première ou montre que l'auteur prend ses distances avec ce qu'elle exprime. D'autant plus lorsque l'enjeu est idéologique comme pour le commerce dans le contexte de la mondialisation, et que, par conséquent, l'impératif de mémorisation de la part du grand public prévaut. Or il est acquis que « l'existence de structures figées favorise la mémorisation de formules » (B. Habert et P. Fiala, 1989 : 95). De fait, celle de défigements de structures figées célèbres aussi.

Si l'on envisage à présent le critère du degré de figement que nous avons tenu à distance jusqu'ici, on pourrait se demander si un fort degré de figement de l'expression première pourrait justement permettre à la seconde de se poser en alternative également forte. A l'inverse, une « expression idiomatique faible » comme le dirait L. Perrin (2011) déterminerait-elle par une sorte de transfert « héréditaire» un faible degré de figement? Il est évident que pour répondre à une telle question, il faudrait disposer de critères sûrs pour définir le degré de figement. B. Lamiroy et J.-R. Klein(2005) en résument la difficulté de la sorte :

«Opacité sémantique ou non-compositionnalité du sens, expressions non motivées, sens figuratif, conventionnalité, préfabrication, restrictions syntaxiques inattendues, blocage des propriétés transformationnelles, blocage de la variation paradigmatique. D'autres critères moins généraux, plus accessoires, sont parfois mentionnés, comme les traces de langue ancienne (ex.chercher noise), la non-actualisation d'un élément (ex. absence du déterminant, plier bagage), les restrictions sélectionnelles (ex. avoir avalé son parapluie) ou l'impossibilité de traduire dans une autre langue (ex. angl. to kick the bucket "mourir" et non pas *renverser le seau)».

Quels figements parmi ceux proposés sont fortement et faiblement idiomatiques ? Sans aller jusqu'à appliquer à nos exemples cette grille de critères afin de les situer sur le continuum, ce qui concentre beaucoup de travaux dans le domaine, nous pourrions y appliquer un autre critère, quantitatif celui-ci, en essayant de préciser combien d'autres défigements s'appuient sur cette séquence figée. Il 
n'existe bien sûr aucun dictionnaire des défigements, et cette démarche ne peut être qu'empirique. Mais celle-ci permettrait de mesurer ce que nous avons appelé le « ratio actuel/virtuel» dans notre dernier ouvrage (2012:47), à savoir le rapport entre le possible déjà réalisé et le possible jamais réalisé du figement en question. Dans cette optique, on pourrait supposer que plus le nombre de défigements d'une expression est important, plus son virtuel s'affaiblit, du fait que les parcours de défigement ont déjà été explorés, et donc, que les possibilités - sans doute les plus pertinentes et ludiques, se sont réduites. En clair, plus le virtuel s'affaiblit - augmentant d'autant l'étendue de l'actuel, moins il y a de chances qu'un défigement très « expressif » apparaisse sur la base d'une expression figée donnée. Passant donc en revue les différentes séquences figées de notre liste, on peut noter les défigements alternatifs suivants :

\begin{tabular}{|l|l|l|l|}
\hline & SF & SF défigées étudiées & SF défigées alternatives \\
\hline a & sur mesure & sur démesure & sur petite mesure \\
\hline $\mathrm{b}$ & poids lourd & poids lourdingue & poids lourdeau; poids très lourd \\
\hline $\mathrm{c}$ & cible mouvante & cible émouvante & cible peu/très mouvante \\
\hline $\mathrm{d}$ & animation offensive & réanimation offensive & animation très offensive ; désanimation offensive \\
\hline $\mathrm{e}$ & commerce équitable & commerce inéquitable & commerce peu/pas très équitable \\
\hline $\mathrm{f}$ & vengeur masqué & vendangeur masqué & vengeur démasqué \\
\hline $\mathrm{g}$ & fête de la bière & défaite de la bière & - \\
\hline $\mathrm{h}$ & trident offensif & strident offensif & trident très offensif \\
\hline $\mathrm{i}$ & père fouettard & ampère fouettard & - \\
\hline
\end{tabular}

(a) ... À Flers, un casting sur (petite) mesure pour Sarkozy(L'Humanité du 07.09.2009).

(b) i. Je lui signale aimablement de regarder les vidéos de Jean Yanne, autrement plus instructives que son numéro de poids lourdeau (Forum sur le site de L'Express $\mathrm{du} 21.08 .2010)$; ii. Teddy Riner en poids très lourd (L'Orient-le-Jour du 03.08.2013) ou Eric Raoult, poids très lourd de la politique (Site Margaud cuisine mais préfère dîner en ville, 12.09.2012)

(c) Sauf que Klitschko n'a aucune envie de se fatiguer et n'a qu'à asséner sa droite massive sur une cible peu mouvante (site Keposport, 04.03.2012); Le code de conduite est une cible très mouvante et très politique (LeTemps.ch, 25.11.2011).

(d) i. Revers de la médaille de l'animation très offensive mise en place par Laurent Blanc...(Leplus.nouvelobs.com, 15.06.2012) ; ii. Désanimation offensive. Après avoir été menés 17-6 à la mi temps, les bleus ont finalement réussi à arracher un match nul face à des irlandais très réalistes (BarDesSports.fr, 06.12.2012).

(e) i Ethique - Lecommerce peu équitable de la grande distribution (Enviro2b.com, 18.05.2011) ; ii. Le café, un commerce pas très équitable (Mediapart.fr, 30.01.2009).

(f) Super W. Bush, le vengeur démasqué (CourrierInternational.fr, 30.08.2007).

(h) José Mourinho utilisera un trident très offensif, ce dimanche 14 août contre Barcelone(Chronofoot.fr, 10.08.2011).

Précisons dès à présent que ces défigements sont tous contenus dans des ordres d'occurrence faibles ; on peut même considérer comme des hapax idiomatiques 
« sur petite mesure», «poids lourdeau » et « désanimation offensive». Les autres, qui résultent souvent (sauf « vengeur démasqué ») de l'intercalation d'un adverbe exprimant l'intensité, sont plus fréquents sans l'être beaucoup : « cible peu mouvante » ( animation peu offensive» n'est pas attesté, pas plus que « trident peu offensif » ou « sur grande mesure ») n' apparaît qu'une dizaine de fois, « trident très offensif » et « animation très offensive » une vingtaine et « cible très mouvante » une quarantaine. Contrairement à ce qu' on pouvait penser, « commerce peu équitable » n'est attesté qu'une soixantaine de fois, soit à peine plus que « commerce pas très équitable » ou « commerce pas équitable ». Enfin, la valeur quantitative de certains défigements est difficile à évaluer, en particulier celle de " poids très lourd» (dans le sens de « personnage imposant») qui subit la forte concurrence des haltères et autres difficultés psychologiques.

Comme on le voit, les défigements de notre corpus semblent être issus de séquences figées peu défigées par ailleurs, ou serait-on tenté de dire, mal défigées, peu efficacement en tout cas : les défigements par intercalation d'adverbes d'intensité semblent ne pas pouvoir lutter ni en termes quantitatifs ni en termes d'expressivité. Si l'on prend le cas du titre de notre article, « commerce inéquitable » prend clairement le dessus sur ses concurrents formés de la sorte. La même remarque vaut pour " sur démesure ", " cible émouvante "; même " poids très lourd ", dans l'état de nos recherches, ne semble pas non plus pouvoir concurrencer « poids lourdingue ». Notons d'ailleurs ici la nette préférence pour le suffixe «-ingue» par rapport à «-eau ».Il est délicat d'induire avec assurance une loi qui explique ces faits. Toutefois, on pourrait se risquer à dire que les défigements produits par 1'ajout de prétendus affixes est jugée plus efficace que l'intercalation d'adverbes d'intensité, peut-être car ce procédé crée un couple de formules (antonymes ou non) plus frappant. Le fait que peu d'autres défigements semblent possibles à partir des séquences figées étudiées laisse entendre que le virtuel desdites séquences est souvent presque épuisé.

\section{En somme}

On pourrait enfin ajouter que les critères de figement de SF défigées ne sont pas fondamentalement différents des critères (comme l'expressivité)qui font qu'une métaphore, par exemple, se fige. Par contre, à l'inverse de la métaphore, dans notre étude demeure une différence, sans doute non décisive mais qui mérite d'être signalée : en effet, étant issu d'une première $\mathrm{SF}$, le nouveau figement ne peut en éliminer la mémoire. Nous voulons dire par là que, du moins pendant un certain temps, celui-ci forme avec la SF originelle un couple imprimant un inévitable dédoublement polyphonique. La qualité et le caractère fonctionnel de ce figement à deux têtes semble alors d'une importance certaine pour la survie du dernier venu. Or l'on sait que « les expressions figées ne sont en effet bien souvent exploitées discursivement que pour les virtualités de défigement de leur sens ascendant, les jeux rhétoriques qui s'y rapportent » (L. Perrin, à paraître). Une expression figée est en clair souvent convoquée pour être manipulée et justement parce qu'elle le permet. La stabilisation du défigement dépend sans doute de sa fréquence d'apparition.

Nous avons dit que le fait qu'il marque une opposition peut s'avérer décisif : est-ce l'effet de l'existence de préfixes courts et expressivement efficaces marquant ce type de relations, ou doit-on penser, a contrario, que c'est le type de relations isolées plus haut qui est décisif ?Le nombre d'exemples envisagé dans cette 
étude ne permet pas de le déterminer. Toutefois, celle-ci aura au moins permis de mettre le doigt sur un aspect des SF peu évoqué, celui des critères qui régissent la potentielle seconde vie des défigements, soit leur lexicalisation.

Enfin, pour répondre à la dernière question que nous nous posions plus haut, le degré de figement pourrait bien se transmettre de la séquence figée à la séquence défigée : c'est le cas pour le degré de figement que l'on qualifiera de moyen (puisqu' on peut au moins y intercaler un adverbe) pour « commerce équitable » et peutêtre « cible mouvante " : en effet, est d'ores et déjà attesté, bien qu'en quantité confidentielle, "commerce très inéquitable » (4 occurrences) mais pas encore « cible peu/très émouvante », dont on peut toutefois aisément prédire l'apparition. Mais cette piste nous conduit plus loin que l'objectif de ce travail, vers le défigement de séquences défigées déjà figées qu'il vaut mieux, nous en conviendrons, réserver à des travaux ultérieurs.

\section{Bibliographie}

ANASTASSIADIS-SYMÉNÉODIS, A. (2003), « Que peut-il arriver à une expression figée ? », in Mejri (coord.) Le figement lexical-Cahiers de lexicologie, 82, Paris : Honoré Champion, 51-59.

Corbin, D. (1988), « Pour un composant lexical associatif et stratifié », in « Lexique, nouveaux modèles » DRLAV 38 Revue de linguistique du Centre de recherche de l'Université Paris VIII, 63-92.

ČERMÁK, F. (1982), Idiomatika a frazeologiečeštiny. Praha : Univerzita Karlova.

Cusimano, C. (2012), La sémantique contemporaine - du sème au thème. Paris : Presses Universitaires de la Sorbonne.

GRÉCIANO, G. (2003), « Le figement s'étend et s'enracine », in Mejri (coord.) Le figement lexical-Cahiers de lexicologie, 82, Paris : Honoré Champion, 41-49.

FiALA, P. et HABERT, B. (1989), « La langue de bois en éclat : les défigements dans les titres de presse quotidienne ", in Mots, 21, Paris : Presses de la Fondation Nationale des sciences politiques, 83-99.

FRADIN, B. (2003), Nouvelles approches en morphologie. Paris : PUF.

GRoss, G. (1996), Les expressions figées en français. Noms composés et autres locutions. Paris : Ophrys.

HAßLER, G. et HÜMMER, C. (2005), « Figement et défigement polylexical : l'effet des modifications dans des locutions figées », in Linx [En ligne], $53 \mid 2005$, en ligne à l'adresse : http ://linx.revues.org/266

KRIEG-PlAnQUe, A. (2012), La notion de "formule » en analyse du discours. Cadre théorique et méthodologique. Besançon : Presses Universitaires de FrancheComté.

- (2009), Analyser les discours institutionnels. Paris : Armand Colin.

LAMIROY, B. et KLEIN, J.-R. (2005), « Le problème central du figement est le semi-figement » dans Linx [En ligne], 53 | 2005, en ligne à l'adresse : http://linx.revues.org/271 
LECLER, A. (2007), " Le défigement : un nouvel indicateur des marques du figement ? », in Cahiers de praxématique 46. Montpellier : Pulm, 43-60.

MEJRI, S. (1998), «La mémoire des séquences figées : une troisième articulation, ou la réhabilitation du culturel dans le linguistique? », in Actes du colloque : La mémoire des mots. Actualité Scientifique, Tunis, AUPELF-UREF, 3-11.

MEL'ČUK, I. (1998), « Collocations and Lexical Functions », in A. P. Cowie (ed.) Phraseology. Theory, Analysis and Applications. Oxford: Clarendon Press, 23-53.

Moon, R. (1998), Fixed Expressions and Idioms in English : A Corpus-based Approach. Oxford : Oxford University Press.

PERRIN, L. (2011), "Figement, énonciation et lexicalisation citative ", in J.C. Anscombre et S. Mejri (éds.), Etudes sur figement : la parole entravée, Paris, Champion, 80-94.

- (à paraître) « Du figement au défigement des unités du lexique », Cahiers de lexicologie, $\mathrm{n}^{\circ} 2013-2$.

RASTIER, F. (1997), " Défigements sémantiques en contexte », in Martins-Baltar, Michel (éd.), La locutionentre langue et usages. Fontenay / Saint Cloud, ENS Editions, 305-329.

SZCZEPANIAK, R. (2006) The Role of Dictionary Use in the Comprehension of Idiom Variants. Tübingen : De Gruyter.

Sullet-Nylander, F. (2005), « Jeux de mots et défigements à la Une de Libération », Langage et société 2/2005 ( $\left.{ }^{\circ} 112\right), 111-139$.

\section{Repères}

\section{Premières pratiques d'écriture: état des recherches francophones}

Numéro coordonné par Jacques David et Marie-France Morin

\section{L'écriture : comment les jeunes élèves l'approchent-ils?}

Contributions de : S. Fraquet, J. David, L. Pulido, F. Lacroix, A. Lainé,

D. Hannouz, M.-F. Morin, P. Nootens, L. Dappe

L'écriture des jeunes élèves : comment les enseignants l'analysent-ils ?

Contributions de : P. Groothuis, F. Mauroux, C. Garcia-Debanc, S. Plane

Renseignements - commandes:

Ecole Normale supérieure de Lyon

Service des éditions - Site Ferdinand Buisson

15 parvis René-Descartes - BP 7000 - 69342 LYON cedex 07

editions@ens-lyon.fr 